Indikationen für chirurgische Eingriffe im Säuglingsalter.

Von

\title{
Walther Usener.
}

(Aus dem Kaiserin Auguste Victoria Hause zur Bekämpfung der Säuglingssterblichkeit im Deutschen Reiche, Charlottenburg. Direkt.: Prof. Dr. Langstein.)

Inhaltsverzeichnis.

Selte

Einleitung . . . . . . . . . . . . . . . . . 73

I. Chirurgische Indikationen . . . . . . . . . . . . 75

II. Pädiatrische Indikationen ${ }^{\circ}$. . . . . . . . . . . . . . . . . . . 78

III. Narkose, Wärmeschutz und Nachbehandlung . . . . . . . . . . . 90

IV. Spezielle Indikationen . . . . . . . . . . . . . . . . . . . . . 95

V. Literaturverzeichnis . . . . . . . . . . . . . . . . . . . . 102

\section{Einleitung.}

Indikationen für chirurgische Eingriffe im Säuglingsalter lassen sich gegenüber den widersprechenden Gesichtspunkten der Chirurgen und Kinderärzte wie für den Erwachsenen nicht anders als von gemeinsamen Grundsätzen aus gewinnen. Als solche können für das Alter des Säuglings und Kleinkindes die Erhaltung und die körperlich, geistig und sozial lebensfähige Entwicklung zugrunde gelegt werden.

Die moderne Chirurgie hat, gestützt auf eine vielseitige wissenschaftliche und technische Arbeit, im Sinne des Heilplans, der operativen Methoden und der Sicherung ihrer Resultate sich mit Erfolg auch den kleinen und zarten Verhältnissen des Säuglingsalters angepaßt. Aber es kann nicht verschwiegen werden, daß diese Erfolge im Vergleich zu den Bestrebungen der Säuglingsfürsorge und der durchgreifenden Bekämpfung der Säuglingssterblichkeit namentlich da begrenzt blieben, wo die Narkose und deren Begleitumstände nicht gleich sorgfältig bedacht und geleitet waren und insbesondere die Vernachlässigung einer sorgfältigen Beurteilung der Gesundheit und Widerstandskraft, ferner der Ernährungsfragen, der Nachbehandlung und Pflege zu unerwarteten Komplikationen und häufigen Mißerfolgen führen mußße, wo also bei aller Höhe chirurgischen Könnens das Resultat infolge einer ungenügenden Erfahrung in den besonderen Lebensbedingungen des wachsenden Kindes gefährdet und zerstört wurde. Daß hier ein exakter Vergleich 
auf Grund einer ausreichenden Statistik chirurgischer Kliniken nicht möglich ist, muß als ein Mangel empfunden werden.

Inzwischen hat die Pädiatrie, ein jüngerer Zweig der Medizin, die Kenntnisse der Physiologie und Pathologie des Säuglingsalters so wesentlich gefördert, daß ihre Mitarbeit in diesen Fragen und ihr Urteil bei der Indikationsstellung gefordert werden kann.

Es besteht ein Widerspruch zwischen der großen Lébensenergie des gesunden Neugeborenen, die ihren Ausdruck findet in der Wachstumsintensität, der Größe des Stoffwechsels, der schnellen und guten Heilung von Wunden, Knochenbrüchen $u$. a. auf der einen Seite und den hohen Zahlen der Säuglingssterblichkeit auf der anderen Seite. Aber dieser Widerspruch ist nur ein scheinbarer. Jedenfalls war es ein verhängnisvoller Irrtum, wenn man glaubte, daß dies die Folge einer natürlichen Auslese sei. Denn' die Faktoren, die Entwicklung und Geschichte des Menschen ausmachen, spielen sich in erster Linie auf dem geistigen Gebiete ab. In der frühen Kindheit besteht aber, abgesehen von dem Zugrundegehen der von Geburt an Lebensunfähigen, schon deswegen nicht das Recht, von Auslese zu reden, weil die soziale Gliederung und die unnatürlichen Bedingungen der Domestikation einer natürlichen Auslese in somatischem Sinn widerstreben. Das junge Kind ist ja von seiner Umgebung völlig abhängig und wenn auch die von Geburt aus weniger widerstandsfähigen Kinder stärker leiden, so können doch ausnahmslos auch die Kräftigeren in gleicher Weise erkranken infolge des verbreiteten Mangels an Kenntnissen der Ernährungsfragen und infolge falscher Pflegegewohnheiten, deren Bekämpfung eine der Hauptaufgaben der Säuglingsfürsorge ist. Und diese Fürsorge muß wie jede hygienische Fürsorge als eine notwendige Korrektur der erwähnten unnatürlichen Bedingungen gewertet werden.

Jener Widerspruch löst sich darin auf, daß die Wachstumsintensität dem Organismus Aufgaben stellt, die nur durch die vollkommene $\mathrm{Zu}$ führung und Verarbeitung der zum Aufbaur notwendigen Stoffe gesichert sind, daß dem höheren Stoffumsatz ein wesentlich höherer Stoffverbrauch und Energieumsatz entspricht, daß die qualitative Zusammensetzung fast noch wichtiger ist wie die notwendige Menge der Zufuhr und daß endlich jeder Eingriff abnormer Art, Störungen der Zufuhr und des Ernährungsablaufs, Krankheit, Infektion und schließlich auch durch seinen Stoffverlust und Mehrbedarf zu regenerativem Aufbau, jeder operative Eingriff eine unzweideutige Störung des normalen Wachstumsvorgangs und eine im Verhältnis zum Erwachsenen viel größere Schädigung bedeutet. 
Man darf sagen, daß die ersten Lebensjahre des Kindes vorwiegend im Zeichen der vegetativen Funktionen stehen und daß die körperliche Entwicklung im allgemeinen je nach dem Grade ihrer Vollkommenheit durch das Wohlbefinden, das sie vermittelt, im günstigen Sinn auf die statischen Funktionen, den Willen und die Entfaltung der psychischen Anlagen einwirkt. Von Auslese kann erst da im Alter des Kleinkindes und Schulkindes die Rede sein, wo denjenigen Kindern, die entweder durch Krankheit zurückgeblieben konstitutionell schwächer oder durch angeborene Defekte und Verbildungen in ihrer Entfaltung gehemmt sind, bei gesunden geistigen Anlagen durch jede Art Störung körperlicher und seelischer Betätigung auch in der Entwicklung geistiger Regsamkeit, in der Ubung der Geschicklichkeit bebindert sind und ihren Altersgenossen nachzustehen beginnen. Diesen Hemmungen gegenüber setzt dann ein weiterer, nicht minder wichtiger Tंeil der Fürsorge in ärztlichem, chirurgisch-orthopädischem und pädagogischem Sinne ein.

Die Tatsache also, daß der Säugling ein wachsender, noch unfertiger Organismus ist, daß er im Verhältnis zu den Neugeborenen der meisten Tierarten in höherem Grade von seiner Umgebung und Pflege abhängig ist, verlangen für seine Erhaltung und Entwicklung den Aufwand sowohl einer großen Arbeit der gesamten Ernährungsfunktionen als auch der Anpassung und Überwindung einer bedeutenden Anzahl Gefährdungen und Krankheiten. Und mit diesen muß auch unabhängig von der günstigeren heilenden Tendenz bei jedem operativen Eingriff gerechnet werden.

\section{Chirurgische Indikationen.}

Zu den allgemeinen Gesichtspunkten, welche die Chirurgie für die Indikationen zu Operationen im frühesten Kindesalter heranzieht, gehört also zunächst die Tatsache, daß die Heiltendenz in diesem Alter eine besonders günstige ist. Es gilt diese Indikation zu möglichst frühzeitigem Operieren besonders für alle die Mißbildungen und Defektbildungen, die aus Wachstumsstörungen hervorgehen. Je jünger ein Individuum ist, um so schneller gehen die Vernarbungsprozesse bei Wunden, Epitheldefekten, Knochenfrakturen, Operationswunden vor sich. Das kann als unbestritten gelten, ist aber an sich kein zureichender Grund zu uneingeschränkter Wahl der frühzeitigen Operation. Er könnte mit irgend einem Vorrecht zunächst nur für solche Òperationen in Betracht kommen, die zugleich als kosmetische erwünscht sind, um so mehr da auch die Narbenbildung zarter und unauffälliger bleibt. 
Denn auch in der späteren Kindheit geht die Heilung noch in annähernd gleich günstiger Weise vonstatten. Es entspricht aber dann weiter dieser heilenden Tendenz des jugendlichen Organismus, die ebenso auch aus der Wachstumsintensität abzuleiten ist, eine formbildende, plastische Tendenz, die der Richtung auf die Formbildung der Art folgt. Wenn Spitzy nur die Tatsache hervorhebt, daß im frühen Kindesalter gleichgroß wie der Wachstumskoeffizient auch der Deformationskoeffizient sei, so scheint mir das, weil einseitig, nicht gerechtfertigt. Wenn man diese an sich unzweifelhafte Tatsache nicht nur auf die Wachstumsintensität, sondern auch auf jene plastisehe Tendenz bezieht, so ist zu unterscheiden, ob dieser formbildende Wachstumstrieb sich, da es sich doch um Pathologisches handelt, auf die normale Gestaltung oder auf eine von ihr abweichende bezieht. Und dann muß eine ausgleichende Tendenz, welche ebenso wie die heilende bestrebt ist, Defekte, Spaltbildungen und dergleichen noch $\mathrm{zu}$ schließen und zu vernarben, unterschieden werden von einer deformierenden, welche eine vorhandene nịcht mehr ausgleichbare Deformität noch zu verstärken neigt. In letzterem Falle geschieht das aber deswegen, weil ein hemmendes Moment, wie es z. B. bei Klumpfuß, Schiefhals n. a. infolge Sehnen- und Muskelverkürzung der Fall ist, die symmetrische Formentwicklung unmöglich macht. Beide Tendenzen sind in der Wachstumsperiode gleich intensiv und gleich beachtenswert. Ganz ebenso wie ein selbst mäßiger Klumpfuß, eine angeborene Hüftluxation, eine durch Muskelverkürzung verursachte geringe Wirbelsäulenneigung unbehandelt zu einer schweren Deformität der Knochen und Gelenkbildung führen kann, ist es nicht mehr zweifelhaft, daß eine Reihe der Fälle der auf fötaler Entwicklungshemmung beruhenden Spaltbildungen wie Nabelbruch, Leistenbruch u. a. völlig verschlossen und ausgeglichen, da $\beta$ auch erworbene rachitische Verbildungen bis zu einem gewissen Grade durch das Wachstum und die statischen Momente selbst korrigiert werden können. Hier handelt es sich also im wesentlichen um Bildungsfehler, die aus einer Hemmung des Verschlusses fötaler Öffnungen und Spalten hervorgehen, von denen man sagen darf, daß der plastische Wachstumstrieb sie, soweit die widerstrebenden Druckverhältnisse therapeutisch überwindbar sind, noch zu schließen vermag. Dort ist durch eine fötale Wachstumsstörung bereits eine Richtung zur Deformierung und asymmetrischen Bildung gegeben. Mit Recht ist für diese letzteren Bildungsfehler eine frühzeitige Behandlung schon lange anerkannt und die Entwicklung der chirurgischen Orthopädie wird immer mehr dahin gehen, sie so frühzeitig wie möglich, damit gleichzeitig auch technisch so wenig eingreifend 
wie möglich und mit besseren Resultaten zu behandeln. Dabei treten aber in vielen Fällen die nicht operativen orthopädischen Methoden an Stelle der blutigen, und gerade hier ist die plastische formbildende Wachstumstendenz als wirksam mitzuhelfen berufen. Durch redressierende Verbände und Behandlung wird dabei Wachstum und Entwicklung nach der normalen Richtung hingelenkt. Es sind dann auch alle Schädigungen an Muskulatur und Gelenken, die bei temporärer Ruhigstellung später eintreten können, zu vermeiden.

Es kann als hierher gehörig noch daran erinnert werden, daß als eine Folge der größeren Wachstumsintensität des jugendlichen Gewebes auch die malignen Geschwülste in der frühen Kindheit in ihrem Verlauf bösartiger sind, wodurch die Prognose ihrer Entfernung eine wesentlich schlechtere wird.

Die frühzeitige Operation wird ferner bei einer Reihe angeborener und erworbener Anomalien deshalb befürwortet, weil durch sie eine bessere körperliche und allgemeine Erziehung gewährleistet ist. Daß z. B. bei kongenitalen Gaumenspalten schon im ersten Lebensjahr so gute Resultate zu erzielen sind, wie sie Hel bing mitgeteilt hat, ist sehr beachtenswert, vorerst aber noch eine Spezialität, die erst mit der Zeit allgemeinere Nachahmung und gleich gute Erfolge zeitigen wird. Eine frühzeitige Operation ist aber natürlich für die ungehemmte Sprachentwicklung entscheidend. Man kann auch sagen, daß die frühzeitige Operation eines Schiefhalses, die frühzeitige Behandlung angeborener oder erworbener Verbildungen an den Beinen eine bessere und freiere Durchführung der körperlichen Erziehung und Entwicklung ermöglicht, so daß dadurch auch die Lebensfähigkeit im sozialen Sinn gesteigert wird. Es scheint mir aber nicht berechtigt, hierher auch, wie Spitzy will, z. B. die Hernien zu rechnen, zum mindesten nicht alle Fälle. Er meint, die Sorge um ein .Größerwerden oder um eine Einklemmung des Bruches halte die Eltern ab, die volle körperliche Erziehung durchzuführen und durch die vielfache Rücksichtnahme werde auch die Erziehung des Willens beschränkt. In manchen dieser Fälle ist die Sorge der Eltern übertrieben und kann beeinflußt werden, in anderen wird man sich mit Rücksicht auf neuropathische Einflüsse nicht einmal zur Operation eines nicht wirklich störenden und großen Bruches entschließen. Bei dem Mastdarmvorfall, wo die Verhältnisse freilich noch etwas anders liegen, da es sich gleichzeitig um die Ubung eines Schließmuskels handelt, wird man im Sinne der Erziehung von einer unnötigen Operation fast immer direkt abraten dürfen.

Schwieriger als für die besprochene frühzeitige Behandlung der, 
angeborenen Verbildungen liegt die Entscheidung bei den Krankheiten der Grenzgebiete, bei den inneren Krankheiten, bei denen der Chirurge wie beim Erwachsenen die Frühoperation, d. h. die Operation im Beginn der Erkrankung zu befürworten geneigt ist. Für das frübeste Kindesalter lassen sich allgemeine Gesichtspunkte nicht aufstellen, und es ist, da es sich um überwiegend schwere Eingriffe handelt, die Frage mabgebend, innerhalb welcher Breite interne Behandlung erfahrungsgemä $\beta$ allein zum Ziele führt.

\section{Pädiatrische Indikationen.}

Die Kinderheilkunde wird in der vorliegenden Frage nicht anders als von der Tatsache der großen Säuglingssterblichkeit ausgehen können und einen erheblichen Teil der Mißerfolge im Verlauf chirurgischer Eingriffe auf die gleichen Ursachen zurückführen müssen, welche jener zugrunde liegen. Ihre allgemeinen Gesichtspunkte lassen sich in der Frage vereinigen, innerhalb welcher Grenzen der Säugling als wachsender Organismus chirurgischen Eingriffen gewachsen ist, eine Frage, welche durch die nahen Beziehungen zwischen Wachstum und Ernährungsvorgang und weiter zwischen Ernährung, Immunität und Konstitution zu beantworten ist.

Die Gesetze des Wachstums sind ihrem eigentlichen Wesen nach noch wenig bekannt. Wir kennen wohl die gesetzmäßige Abfolge des Gewichts, der proportionalen Entwicklung der Länge und der Form, die chemischen Elemente des Aufbaus und wichtige dynamische Gesetze desselben. Wie aber der Organismus aus der natürlichen und besonders aus der künstlichen Nahrung den biochemischen Aufbau leistet, wissen wir nicht. Das gilt in erster Linie von der Struktur und dem Stoffwechsel der lebendigen Eiweißkörper. Da aber die den Organismus aufbauenden Elemente bekannt sind, so ist es wenigstens möglich, durch das Studium ihres Verhältnisses zueinander und der Ursachen der Ernährungskrankheiten $\mathrm{zu}$ Vorstellungen über Wachstumsstörungen zu gelangen, die für das Verständnis und für das ärztliche Handeln fruchtbar geworden sind. Das wäre aber nicht möglich ohne die Vertiefung der neveren klinischen Forschungen über die feineren physiologisehen Verhältnisse des gesunden und kranken Säuglings und die Symptome seines körperlichen und seelischen Zustandes in allen Phasen seiner Entwicklung. Während die Gewichtkurve vorerst der sinnfälligste Anhaltspunkt für das Wachstum der Maße, ferner das Verhältnis des Gewichts zu Länge und Proportion derjenige für die normale Formentwicklung ist. zeigt erst die klinische Beobachtung und Beurteilung des Ernährungs- 
zustandes, der körperlichen und psychisehen Gesundheit in der gesamten Entwicklung den sicheren Maßstab für die normale Qualität des Wachstums an. In dieser Betrachtungsweise liegt einer der Fortschritte der neueren pädratrischen Forschung für die früheste Kindheit.

Wirklich gute Ernährungsbedingungen leistet erfahrungsgemä $B$ nur die Muttermilch. Weshalb das so ist, ob besondere noch unbekannte Bestandteile, wie etwa die Wirkung von in der Frauenmilch vorhandenen oder vorgebildeten Katalysatoren (Czerny) mitspielen, ist nicht entschieden. Gegenüber dem Studium einzelner Komponenten, das in der Frage keine Lösung gebracht hat, wies Langstein auf die Bedeutung der Korrelation hin, in der die einzelnen Elemente arteigen zueinander stehen. Man darf sich vorstellen, daß gerade dies arteigene Verhältnis der als Baumaterial bedeutungsvollen anorganischen zu den organischen Stoffen einen bestimmenden Einfluß auf den Aufbau und die Bindungsverhältnisse im Protoplasma ausübt. ' Und das um so mehr, da auch die Verarbeitung und Umwandlung im Darm infolge einer ungestörten Anpassung der Verdaunngsarbeit, infolge einer einheitlichen Darmflora innerhalb gewisser Grenzen gesetzmäßig vor sich gehen kann. Bei der artfremden Nahrung ist die Korrelation gestört, der Chemismus der Verdauungsfermente und die Bakterienflora in wechselnder Art auf sie eingestellt. In einem ähnlichen Sinn hat Finkelstein Wert aù die Unterschiede in der Zusammensetzung der arteigenen und artfremden Molke gelegt.

Während dem Wachstumstrieb der Art folgend Längen- und Proportionenwachstum ungehemmt bis an die Grenze der vorhandenen Reserven weitergehen, erleidet der gesamte Vorgang der Frnährung und des Massenwachstums Störungen dann, wenn ein Mißverhältnis besteht zwischen dem Angebot an Nahrung und der Fähigkeit, angebotene mehr weniger artfremde Nahrung zu verarbeiten. Als Ursachen solcher Störungen kommen in Betracht einerseits Uberernährung und Unterernährung besonders qualitativer Art, anderseits alle jene Momente, die als krankhafte besonders infektiöse Prozesse oder als konstitutionelle Abartungen die Fähigkeit des Organismus einschränken, die aufgenommene Nahrung 'zum Aufbau auszunutzen. So stehen bei dem wachsenden Kinde Emährungsvorgang, Immunität und Konstitution in fortwährender Wechselbeziehung. Die künstliche Ernährung erfordert auch von dem vollwertigen Kinde unter allen Umständen eine Mehrleistung und besondere Anpassungsarbeit, bei dem kranken Kinde kann diese je nach der Güte der hygienischen Verhältnisse, der Qualität der Nahrung, der Pflege und nach dem konstitutionellen Zustand eine bedeutende werden. 
Der chirurgische Eingriff und seine Begleitumstände stehen zu dem Vorgang der Ernährung und zu der Ernährungsstörungen in einer doppelten Beziehung. Einerseits bedeutet die gesetzte Wunde einen gewissen Verlust an Substanz, es wird zur Reparation eine Mehrleistung der aufbauenden und regenerativen Funktion und dazu eine besondere Summe an Baustoffen neben dem laufenden Verbrauchsmaß notwendig, anderseits kanh durch den operativen Eingriff dort, wo er einen in seinem Ernährungszustand gestörten, durch infektiöse Prozesse in seiner Verdauungsfunktion und in seinem Widerstand geschwächten Körper trifft, der sonst noch vermeidbare Zusammenbruch eingeleitet werden. Für das ernährungskranke Kind entsteht eine zweifellose Schädigung, die nur vergleichbar ist derjenigen, die eine akut hinzutretende Magendarmerkrankung oder eine Infektion auslösen würde. Denn zu der schon vorhandenen negativen Bilanz der zum Aufbau notwendigen Substanzen, die durch geeignete wiederaufbauende Ernährungsbehandlung zu reparieren wäre, tritt ein plötzlicher Mehrverlust und noch eine zur Regeneration gebrauchte fortlaufende Mehrbelastung der gesamten Ernährungsfunktion. Und das ist um so mehr zu fürchten, wenn ärztliche Überwachung und Pflege nach der Operation dem Ernst der Lage nicht gewachsen ist, wenn auch noch Hunger oder Verdurstung infolge Appetitlosigkeit oder therapeutisoher Maßnahmen den Zusammenbruch des Stoffwechsels beschleunigen.

Alle Störungen des Ernährungsvorganges, mögen sie von Unteroder Überernährung ausgehen, laufen ja letzten Endes hinaus auf eine Schädigung des wachsenden Körpers an seinem notwendigen Baumaterial. Es kann hier nur kurz an Bekanntes erinnert werden. Neben den durch einseitige Ernährung und auch Uberemährung entstehenden Stoffweehselstörungen infolge Unterbilanz an Salzen, Stickstoff, Fett oder Kohlenhydraten sei die auch experimentell erforschte schwere Schädigung durch Flüssigkeitsmangel hervorgehoben. Der Säugling wird in seiner Lebensfähigkeit nicht nur ungleich früher und schwerer durch Wassermangel beeinträchtigt als der Erwachsene, sondern es ist auch bei Hungerkost sein Flüssigkeitsbedürfnis gesteigert. Grund dazu ist der größere Wasserreichtum der Gewebe, dem eine höhere Wasserdurchspülung entspricht. Da ferner die Stoffwechselvorgänge an die Anwesenheit von Wasser gebunden sind und dér Säugling zunächst durch gesteigerte oft starke motorische Unruhe beim Hunger reagiert, so ist auch dadurch der Wasserbedarf erhöht. Ein gleiches kann dann der Fall sein, wenn nach opérativen Eingriffen infolge Unbehagens der Lagerung, Fesselung oder Schmerz die Motilität gesteigert ist. 
Ähnlich schwer wie Wassermangel wirken nach experimenteller und klinischer Erfahrung Mangel an Salz und Zucker in der Nahrung des wachsenden Organismus.

Für alle Ernährungsstörungen gilt ferner, daß in ihren Anfängen, zu einer Zeit wo dem Ungeübten noch keine deutlichen Folgen und Krankheitserscheinungen sich aufdrängen, bereits der Boden für schwerere Störungen vorbereitet ist. Die initialen Symptome sind neben der Anamnese also besonders wichtig.

Es gehört demnach die Beurteilung des Ernährungszustandes, die peinliche anamnestische und. klinische Feststellung jeder auch beginnenden Ernährungsstörung zu den Grundlagen, welche die Indikationen zu einer Operation im Säuglingsalter mitbestimmen müssen. In dringlichen Fällen bei schon vorhandener Ernährungsstörung bildet diese Diagnose die Grundlage des gesamten Heilplans, der die Wahl und den Zeitpunkt des operativen Eingriffs mitbeurteilt und die Ernährung und Pflege so regelt, daß die Klippen zwischen Inanition und Verschlimmerung der Emährungsstörung vermieden werden.

Mit dem Ernährungszustand hängt der Bestand der natürlichen Immunität aufs engste zusammen. In dem Augenblick, wo die Indikationen zu einem operativen Eingriff erörtert werden, ist neben dem Ernährungszustand nichts so bedeutungsvoll wie die Frage, ob die Immunität des Kindes ausreicht, wie sie bei vorhandenen infektiösen Prozessen in ihrem Bestand zu erhalten oder zu heben möglich ist. Denn bei jedem operativen Eingriff werden die Schutzkräftè des Körpers mobil gemacht, ihr Bestand angegriffen, und wenn schon eine Infektion vorliegt, unter Umständen aufgebraucht. Die Reserven des wachsenden Organismus sind aber an sich nicht groß. Mit jeder tiefergreifenden Störung des Ernährungsvorganges erlischt aber zunehmend die Fähigkeit der Antikörperbildung und der zur Heilung von Wunden und infektiösen Vorgängen notwendigen Regenerationsleistung. Das ist nur dadurch möglich, daß erhebliche Störungen der Zellfunktion entstanden sind und daß diejenigen Reserven, die zum Ausgleich von Verlusten eintreten sollten, erschöpft sind.

Eine Erschöpfung der Reserven unter schwereren Störungen des Ernährungsablaufes ist ohne weiteres verständlich beim wachsenden Organismus. Schwieriger ist es, sich den Vorgang und das Wesen der Störung der Zellfunktion vorzustellen. Denkt man dabei zunächst an die Schwächung der Muskulatur des Skeletts und des Zirkulations. apparates oder an die Herabsetzung der Sekretionstätigkeit der Drüsen, 
so mag an die auch experimentell gesicherten Tatsachen unter Inanition, an die allgemeine Muskelschwäche, Zirkulationsschwäche und an die Herabsetzung der Salzsäureproduktion im Magen erinnert werden. Die letztere ist auch für Infektionen allein sichergestellt (Grünfelder) und macht die Appetitlosigkeit vieler unter infektiösen Prozessen operierter Säuglinge und die dadurch oft bedingten schweren Schädigungen verständlich. Damit werden aber die antitoxischen Reaktionserscheinungen und die Schwächung der hierbei beteiligten Zellfunktionen durch Ernährungsstörung noch nicht befriedigend erklärt. Es bestehen jedenfalls nach Erfahrung und Experiment nahe Beziehungen zwischen Ernährung und natürlicher Immunität; durch Uberernährung wie Unterernährung wird ihr Bestand vermindert. Qualitative Unterernährung insbesondere an Wasser und Salzen schädigt den wachsenden Körper stärker als absoluter Hunger. Anderseits wirkt jede Infektion beim Säugling auf den Ernährungszustand zurück. Nur das Brustkind bildet innerhalb gewisser namentlich durch seine Konstitution gegebener Grenzen eine Ausnahme.

P. Ehrlich hat damit, daß er auf Grund seiner Vorstellung über die immunisatorischen Vorgänge die Anschauung einer Analogie bzw. Identität der assimilatorischen und der Immunorezeptoren ausspricht, den Weg zum Verständnis gewiesen. Wie unter schweren und septischen Infektionen die immunisatorische Kraft des gesamten Körpers, wie lokal die entzündlichen und regenerativen Funktionen der Zelle erlahmen, weil die vorhandenen Immunorezeptoren der tberzahl der Toxine nicht gewachsen sind, so erlischt bei den Stoffwechselstörungen der qualitativen Inanition diese Fähigkeit, weil die für Bildung solcher Rezeptoren erforderlichen Reserven erschöpft sind, während die vorhandenen Rezeptoren für die Assimilationsprozesse und bestimmte Kategorien der Ernährungsreserven schon kaum mehr genügen. So kommt es nach einer Operation zn ganz auffallend verzögerter Heilung, zu torpiden Granulationen, zum Ausbleiben jedes gesunden Vorgangs der Reaktion und Regeneration und auch oft genug zum beschleunigten tödlichen Verfall.

Es bilden diese Beziehungen der Immunität zum Emährungsvorgang einen weiteren eindringlichen Grund zu der Forderung, daß Säuglinge mit Ernährungsstörungen jeden Grades, auch den verdeckten und anscheinend leichten chronischen oder nach kurz rorher überstandener Ernährungsstörung irgendwelchen Operationen nicht unterzogen werden sollten und daß bei allen Infektionen, die dringliche Eingriffe erfordern, die Verordnung und C'berwachung der Ernährung mit der 
größten Sorgfalt geleitet und als ein wesentlicher Teil der Behandlung angesehen und durchgeführt werden muß. Ohne Sicherstellung einer optimalen, in vielen Fällen der natürlichen Ernährung, und einer kundigen Pflege ist der Erfoly von romherein in Frage gestellt. Das gilt um so mehr, als beim anfälligeren Kinde infektiöse Erkrankungen und gerade auch solche. die ein operatives Eingreifen erfordern, die Neigung zu sekundären Magendarmerkrankungen bedingen. da bei herabgesetzter Toleranz die Grenze zwischen ungenügender und zu reichlicher Ernährung sorgfältig nach dem einzelnen Fall zu ziehen und leicht zu verfehlen ist. Und noch ein drittes; es gibt eine Reihe infektiöser Prozesse, wie die Pyodermien, gewisse Formen des Empyems, der Otitis media u. a. Erkrankungen des Grenzgebietes, von denen wir wissen, daß sie nichts anderes sind als der Ausdruck einer durch chronische Ernährungsstörungen gesunkenen Immunität: sie gehören unzweifelhaft in das Gebiet des Pädiaters, denn die Hebung der Widerstandskraft durch die Wiederherstellung des geschädigten Ernährungsbestandes ist hier die Grundlage der Therapie, der gegenüber unter Umständen jeder größere chirurgische Eingriff unnötig wird.

Es soll hier nur kurn die Frage gestreift werden, welche Komponenten der Nahrung die Immunität begünstigen und das Fehlen welcher Bestandteile sie srhädigen. weil die Anamnese in manchen Fällen schon einen warnenden Fingerzeig bieten kann. Und da muß gesagt werden, daß, wenn auch beachtenswerte experimentelle Untersuchungen von Weigert, die Bedeutung des Fettes und weitere Versuche von Tho mas und Horn "mann die Bedlentung des Eiweißes für das Vorhandensein reichlicher Srhutzstrffe bei den Versuchstieren wahrscheinlich machen, sich doch ein praktischer Fortschritt daraus nicht ergibt. Wichtiger ist die klinische Erfahrung und die negative Seite ihrer Resultate, daß jede einseitige Ernährmg hesrnders beim wachsenden Organismus für den Bextand der I mmmmitat rerhängnisroll ist. Praktisch ist danach jede einseitige Ernährung schon nach der Anamnese. besonders länger dauernde a usschlielliche orler überreichliche Milchfütterung, oder einseitige Mehlernährung eine Kontraindikation für t'bereilung jeder nicht dringlichen und aufschiehbaren Operation.

Neben Ernährung und Immnnität spielen die für die Beurteilung des Kindes besonders wichtigen Tatsachen der angeborenen Anlagen, der Konstitution und der Diathesen eine Rolle. Jede abnorme Konstitution muß gewertet werden als eine Erschwerung jener Anpassungsarbeit, die der von seiner Umgebung in allen Lebensbedingungen abhängige junge Organismus neben seiner Wachstumsfunktion zu leisten 
hat. Der Ausgleich zwischen mangelhafter Anlage und äußeren Lebensverhältnissen kann je nach der Größe des Defektes und der Güte und ZweckmäBigkeit der Nahrung und Pflege früher oder später im Verlauf der Kindheit sich vollziehen. Der Kinderarzt ist mit Recht geneigt, bei vielen dieser konstitutionell abnormen und dadurch oft schwergefährdeten Kinder von jeder nicht notwendigen Operation abzuraten und bei dringlichen Eingriffen je nach dem Zustand des Kindes Bedingungen für den Heilplan zu stellen. Sicher ist, daß ebensowenig wie irgend eine künstliche Nährmischung von einer Reihe günstiger Erfolge her ohne weiteres als eine gute Nahrung für jedes Kind gelten kann, es erlaubt sein darf, eine für das vollwertige Kind berechtigte Operationsindikation auf das konstitutionell abnorme Kind zu übertragen. Es sind dabej zwei Momente zu berücksichtigen. Einmal haben alle Konstitutionsanomalien Beziehungen zu Ernährungszustand und Wachstumsvorgang und zweitens handelt es sich um mannigfache krankhafte Symptome, die sowohl jeden anderen Krankheitsverlauf wie auch den Operations- und Heilungsverlauf ungünstig beeinflussen können.

Schon bei einer Reihe von Kindern, die nicht das Bild fest umgrenzter konstitutioneller Abartungen darbieten, ist bekannt, daß sie in ihrem Verhalten von der Norm abweichen. So die Säuglinge, die bei überwiegender Mehlnahrung oder Milchfütterung schlecht, bei entsprechenden Zusätzen besser gedeihen, während doch andere diese fettärmeren oder kohlenhydratärmeren Nahrungsgemische gut ausnutzen. Es gibt Kinder mit anämischen Zuständen, deren Eisendepots früher als normal erschöpft sind, andere, deren Kalkdepots frübzeitig ungenügend werden, so daß sie ber sonst günstigen Bedingungen bald Zeichen von Rachitis oder Spasmophilie aufweisen. Beides ist in sinnfälliger Weise bei den Frühgeburten der Fall. Die Erfahrungen der Frühgeburtenstation des Kaiserín Auguste Victoria Hauses und die klinischen Untersuchungen lehren, daß man ein Recht hat, Frühgeburten im dringlichen Fall zu operieren und sie nicht als lebensunfähig aufzugeben, allerdings nur dort, wo alle Bedingungen der Pflege darauf eingestellt werden können, wo die Technik beherrscht wird, wo das Wärmebedürfnis durch entsprechende Einrichtungen berücksichtigt werden kann und natürliche Ernährung erreichbar ist.

Als eine zweite Reihe von Erkrankungen, die ätiologisch teilweise auf angeborener Anlage beruhe, sind Barlowsche Krankheit, Rachitis und Spasmophilie zu nennen. Erstere hat abgesehen von kaum ver- 
zeihlichen diagnostischen Irttümern für diesen Zusammenhang keine Bedeutung. Um so mehr die Rachitis und Spasmophilie. Beide hängen häufig mit Fehlern der Ernährung zusammen, abgesehen von ihrer konstitutionellen Komponente. Je früher bei beiden die ersten Zeichen auftreten, um so ernster ist die Anlage zu bewerten, um so dringender die Aufgabe der Ernährung und Pflege. Es sei erwähnt, wie schwer die Rachitis auf der Höhe der Erkrankung Motilität, Agilität und Immunität des Kindes beeinflussen und wie die Erkrankung des Thorax auf die Funktion der Brust- und Bauchorgane einwirkt. Der gesamte Heilplan kann zunächst auf längere Zeit nur gerichtet sein auf die mitverursachende Ernährungsstörung, auf die Pflege und Ausbildung der Muskulatur und die Verhütung schwerer Schädigungen. Erst später kann an eine etwa dann noch notwendige orthopädische oder chirurgische Korrektur gedacht werden. Auch andere nicht dringende operative Eingriffe müssen während des floriden Stadiums ausgeschlossen bleiben.

Die Spasmophilie bleibt häufig latent, ihre Manifestationen können aber durch akute alimentäre oder infektiöse Erkrankungen, wahrscheinlich auch durch das Trauma operativer Eingriffe ausgelöst werden, wenn auch die Narkose vorübergehend entgegengesetzt wirken muß. Deshalb ist die Erkennung des latenten Zustandes namentlich in der kritischen Zeit vor dem Entschluß zu einer Operation zu beachten. Dort wo dringliche Operationen vorliegen, ist dann die kunstgerechte Kalziumtherapie (Blühdorn, Göppert) sofort einzuleiten, da durch diese jede schädliche Hungerdiät überflüssig wird.

Von den eigentlichen Diathesen besteht bei der exsudativen Diathese (Czerny) eine erhöhte Vulnerabilität der Haut und Schleimhäute, es kommt zu ungewöhnlichen Reaktionen der Epitheldecke, der oberflächlichen Blut- und Lymphbahnen mit einer starken Neigung zu sekundären oberflächlichen und tiefergreifenden Infektionen, wie Pyodermie, Erysipel, Phlegmone und Drüsenentzündungen. Je nach dem Grade der Diathese sind diese Kinder also bei jedem chirurgischen Eingriff gefährdet und Infektionen in erhöhtem Maße ausgesetzt. Sorgfältige Pflege der Haut und des ganzen Kindes ist notwendig vor und nach jeder Operation. Gleich beachtenswert für chirurgische Stationen ist auch der Schutz solcher Kinder vor katarrhalischen Infektionen und Infektionskrankheiten, die als Komplikationẹn nach der Operation zu fürchten sind.

Die Neuropathie bedeutet eine Steigerung der sensiblen Reizbarkeit und der nervösen (motorischen und sekretorischen) und psychischen 
Reaktionen. Abnormer Hunger oder Appetitlosigkeit können Symptome sein, die nur durch große Aufmerksamkeit der ärztlichen Leitung und Hingabe der Pflege zu überwinden sind oder den Erfolg in Frage stellen. Die Fremdartigkeit der Umgebung kann diese krankhaften Reaktionen erheblich steigern, sie allerdings bisweilen auch günstig beeinflussen. Durch alles dieses kann der Ernährungszustand in Gefahr geraten und auch Herz und Kreislauf leiden. Jede Polypragmasie der kleinen Chirurgie ist $\mathrm{zu}$ vermeiden. Bei notwendigen Operationen sind besondere Beruhigungsmittel in der Nachperiode oft unentbehrlich, wobei besonders Urethan in entsprechend hohen Dosen (Bertling [bei Göppert]), auch Brom oder. Chloral zu empfehlen sind.

Die dargelegten pädiatrischen Gesichtspunkte lassen sich nunmehr dahin zusammenfassen, da $B$ eine Berechtigung za frühzeitigen Operationen im Sinne der Chirurgen in allen dringlichen Fällen nur dann gegeben ist, wenn günstige Bedingungen des Ernährungszustandes, der Immunität und Konstitution durch sorgfältige Diagnose erwiesen sind und in den dringlichen lebenswichtigen Fällen dann, wenn durch die bestmöglichen Ernährungs- und Pflegebedingungen und pädiatrische Behandlung der Erfolg unterstützt und gesichert ist.

Nicht allein für den letzteren Fall, sondern für jeden Fall wäre die Forderung am besten dort erfüllt, wo an Kinder- und Säuglingskliniken chirurgische Abteilungen angeschlossen sind, in denen auch der Kinderarzt beratend mitarbeitet und eine in seinem Sinne gut ausgebildete Schwester Beobachtung und Pflege in Händen hat. Gleich günstige Verhältnisse liegen dort vor, wo ein speziell interessierter Chirurge in einem Säuglingsheim operieren und das Kind in der Behandlung des Pädiaters verbleiben kann. Für alle anderen Fälle wäre, wo es möglich gemacht werden kann, zu empfehlen, daß Säuglinge, namentlich ernährungsgestörte und sonst gefährdete, nach der Operation unter günstigen Transportbedingungen einem Säuglingsheim überwiesen werden.

Die Tatsache verdient jedenfalls hervorgehoben zu werden, daß die Diagnose Gesundheit im Säuglingsalter, insbesondere dort, wo es sich um die Beurteilung der Frage handelt, ob eine wünschenswerte aber nicht dringliche Operation vorgenommen werden soll, wesentlich schwieriger ist als im späteren Alter. Es darf kein $Z$ weifel bleiben, daß diese Frage in jedem Fall zu stellen ist; denn es handelt sich in erster Linie immer um die Erhaltung des Kindes.

Der Erwachsene, der in seiner körperlichen Entwicklung ausgereift 
und den Lebensbedingungen innerhalb der, seiner Anlage nach, möglichen Grenzen angepaßt ist, kanñ in dem Sinne, der hier in Frage steht, als gesund gelten, wenn er normal gebildete und funktionierende innere Organe hat. Es sind denn auch hier die gleichartigen kontraindizierenden kongenitalen Fehler im Säuglingsalter, wie Herzfehler, Lungenatelektase, Stenosen der Atem- und Verdauungswege $\mathbf{u}$. a. nicht in den Bereich der Besprechung gezogen, weil sie jedem Arzt in dieser Hinsicht geläufig sind. Das junge Kind dagegen ist in seinem Wachstum und in seiner Entwicklung unfertig. es ist ungewiß. wie es die Aufgaben der Wachstumsfunktion und die Anpassungsarbeit nach Maßgabe seiner noch unbestimmten Konstitution leisten wird. Es kann da die Grenze des Gesunden nicht scharf geñug gefaßt werden. Die Entscheidung kann nur dahin lauten, daß in erster Linje an Ernährungszustand und Immunität die strengsten Apforderungen gestellt werden und daß die konstitutionellen Verhältnisse abzuwägen sind; erst in zweiter Linie kann gefragt werden, ob die durch eine Verschiebung auf spätere Zeit entstehenden Nachteile größere sind, als das Wagnis einer eingreifendén Operation unter ungünstigen Bedingungen.

Von chirurgischer Seite hat Spitzy den Versuch gemacht, bestimmte Regeln aufzustellen; nach denen die Gesundheit zu beurteilen und die Ubernahme einer Operation zu bestimmen ist:

1. Fin Neugeborener soll nur operiert werden, wenn sein Gewicht über $3000 \mathrm{~g}$ beträgt. (Natürlich macht die Indicatio vitalis eine Ausnahme.)

Bei älteren Säuglingen halte man sich an die entsprechenden Verhältniszahlen und berücksichtige die übrigen Indikatoren des Frnährungszustandes. Wenn möglich, verschiebe man die Operation eventuell in eine günstigere Ernährungsphase.

2. Man operiere niemals in einer absteigenden Gewichtskurve. Wenige Beobachtungstage genügen.

Das bedeutet einen erheblichen Fortschritt gegenüber einem in dieser Hinsicht indikationslosen Operieren. Indessen können diese Regeln nicht als ausreichenk golten. Denn für ein Neugeborenes gibt es nur sine, die Indicatio vitalis: und für den älteren säng̣ling genügen proportionales Gewicht rnd Berickxichigung der Indikatoren des Emährungszostandes nur dann im Zweifelshll, wenn eine mehr weniger lange Beobachtungszeit die Vollwertigkeit des letzteren, eine gute Immunität und das Fehlen bectenklicher Konstitutionsmängel erwiesen hat. Kaum weniger gefährlich wie clie absteigende Gewichtskurve ist die trügerisch aufsteigende, wie bei kohlenhydratreicher Kost. 
Gleichfalls von chirurgischer Tätigkeit her suchte ich schon vor Jahren die nachfolgenden Gesichtspunkte zu gewinnen, die im wesentlichen auf die Ernährungsfrage, die Infektionsverhütung und erst tastend auf die damals noch nicht durchgearbeiteten konstitutionellen Fragen gerichtet waren:

1. Für die Beurteilung der Ernährung war Anamnese und vor allem eine länger dauernde regelmäßige Beobachtung grundlegend.

Brustkinder wurden nur dann zur Operation aufgenommen, wenn die Möglichkeit, das Kind im Krankenhaus weiterzustillen, durch die Beobachtung erwiesen war. Bei zuverlässigen häuslichen Verhältnissen erwies sich häufig etwa vom dritten Tag ab ambulante Weiterbehandlung als gut durchführbar.

Von den Flaschenkindern wurden zunächst diejenigen auf unbestimmt zurückgestellt, in deren Anamnese Ernährungsstörungen, Durchfälle, ferner einseitige Milch- oder Mehlernährung angegeben war. Diese wurden auf eine dem Alter entsprechende Nahrung umgesetzt nach Maßgabe der Budin-Heubnerschen Zahlen. Sie wurden ebenso wie andere annähernd normal ernährte und entwickelte Säuglinge über einige ( $3-6)$ Wochen bezüglich ihrer Zunahme und Gesundheit beobachtet wobei auf Rachitis, Infektionen, Haut und psychisches Verhalten geachtet wurde. Erst wenn die ambulante Beobachtung keinen Grund mehr zur Ablehnung gab, wurden sie klinisch aufgenommen, aber in jedem Fall erst 3-6 Tage bei gleichel Kost und Wägung kontrolliert, ehe die Vornahme der Operation den Eltern zugesagt wurde. Einigemal war ich auch dann noch gezwungen, wieder zu entlassen und den gewünschten Eingritf zu verschieben.

Säuglinge im ersten Lebensvierteljahr wurden nur zu lebensnotwendigen Operationen aufgenommen.

2. Auf die Neigung zu Erkrankung der Haut und Infektionen der Haut und Schleimhäute wurde besonders geachtet. Dazu wurde auch die Prophylaxe der Infektionskrankheiten durch genaue Anamnese berücksichtigt und in Epidemiezeiten die Operation lieber verschoben.

Diese Bedingungen, wenn schon strenger als die von Spitzy später formulierten, enthalten doch noch keineswegs alles das, was wir heute fordern müssen, besonders nicht die eingehendere Berücksichtigung der konstitutionellen Momente und die feinere Beurteilung nach klinischen Symptomen, wie im vorstehenden in den Grundzügen dargelegt wurde.

Es gehört zu der Stärke und dem praktischen Wert des Finkelsteinschen Systems, daß es, von der Einheit des Ernähungsvorgangs und seiner Beziehung zu Wachstum und normaler Entwicklung ans- 
gehend, das gesamte klinische Verhalten in den Mittelpunkt der Diagnostik, des Ernährungszustandes und der Gesundheit stellt. Gleichzeitig ist auf die grundlegende Bedeutung der Cernyschen Betrachtungsweise für die Erkenntnis und den Stoffwechsel isolierter Nährschäden und der Diathesen zu verweisen. Für die Klinik ist die Beurteilung des Gesamtzustandes des Kindes und seiner Reaktion auf Ernährung und Umgebung das Wesentliche. Dazu ist einmalige Untersuchung nicht in allen Fällen ausreichend, in zweifelhaften Fällen meiner Uberzeugung nach vor Ubernahme eines chirurgischen Eingriffs eine zeitweise Beobachtung grundsätzlich zu empfehlen.

Die allgemeinen pädiatrischen Indikationen können abschließend durch etwa folgende Forderungen zusammengefaßt werden:

Unbeschadet der berechtigten chirurgischen Gesichtspunkte zur frühzeitigen Operation ist für den Pädiater die Erhaltung der optimalen Lebens- und Wachstumsbedingungen grundlegend.

Ein Säugling soll danach nicht dringlichen Operationen nur unterzogen werden jenseits des ersten Lebensvierteljahrs bei normalem Ernährungszustand und guten Ernährungsbedingungen, bei gutem Zustand der Immunität, bei Abwesenheit kontraindizierender konstitutioneller Erkrankung.

An die Diagnose Gesundheit sollten strenge Anforderungen gestellt werden, insbesondere sollte sie stets an eine den Verhältnissen entsprechende Beobachtung gebunden sein.

In allen dringlichen Fällen ist neben dem zur Erhaltung des Lebens erforderlichen Eingriff die pädiatrische Beurteilung des Gesundheitszustandes und wo notwendig, frühzeitige Behandlung zu erstreben. Sachkundige Pflege jst auf chirurgischen Stationen für Säuglinge unbedingt zu fordern. Ein weiterer Weg ist die Angliederung chirurgischer Tätigkeit an bestehende Säuglingsheime oder die Uberweisung operierter, behandlungsbedürftiger Säuglinge an ein solches Heim.

Sowohl die Stärkung der natürlichen Immunität durch Ernährung und Pflege, wie die Verhütung von komplizierenden Infektionen, ganz besonders bei konstitutionell Gefährdeten, ist mit allen Mitteln zu unterstützen. 


\section{Narkose, Wärmeschutz und Nachbehandlung.}

Es bleibt nunmehr von allgemeinen Gesichtspunkten noch die Besprechung der Frage, inwieweit das Kreislaufsystem de Säuglings den Einwirkungen der Narkose und Operation gewachsen ist. Die Entscheidung dieser Frage ist von Chirurgen wie vom Kinderarzt gleichermaßen nach seiner Erfahrung zu treffen.

Von pädiatrischer Seite hat Thjemich seine Anschauung über die Anpassungsfähigkeit des jugendlichen Kreislaufs dahin zusammengefaßst. daß auf Grund der Besonderheiten, wie der geringen Widerstände für die Herzarbeit infolge der Weite der Ventrikel, der Ostien und Arterienquerschnitte und des entsprechend niederen Blutdrucks einerseits und der Unverbrauchtheit des durch Gifte wie Alkohol und Nikotin und durch chronische rezidivierende Infektionen nicht geschädigten Kreislaufsystems ,das kindliche Herz und GefäBsystem in ganz ausgezeichneter Weise plötzlich gesteigerten Anforderungen an seime Leistung zu genügen und erhebliche Zirkulationsstörungen lange Zeit zu überwinden vermag".

Ich kann mich dieser Anschauung, wenigstens für das Gefäßsystem, nicht vorbehaltlos anschließen. Sicher entspricht das normale Herzund Gefäßsystem des Kindes in hohem Maße den Anforderungen des Wachstums. Das Herzgewicht im Verhältnis zum Körpergewicht ist, was als Maßstab für die Größe der Leistungsfähigkeit des Herzens gelten kann, beim Neugeborenen am höchsten, sinkt vorübergehend im zweiten Jahr und nimmt von da $\mathrm{ab}$ fortschreitend $\mathrm{zu}$. $\mathrm{Zu}$ dieser Zeit stellt der Ubergang von der liegenden zur aufrechten Haltung, der Úbergang zu freier Fortbewegung an die Anpassungsfähigkeit des Herzens neue Anfgaben. Ebenfalls entsteht dureh die 'Umbildung der Gestaltung des Thorax und die starke (vierfache) Vergrößerung des Atemvolumens anch von seiten der Lunge dem Herzen ein Arbeitszuwachs. Alle diese Aufgaben erfüllt das gesunde Herz ohne Störung. Es kommen dabei freilich extremere körperliche Arbeitsleistungen nicht vor, wie etwa bei Kindern im Schwlalter und in der Entwicklungszeit, denen gegenüber das Herz sich durch I'bung anpassen muB. Der gesamte Umbildungsvorgang rollzieht sich vielmehr allmählich. er ist in weiten Grenzen von dem Triebleben des Kindes. von Agilität und psychischer Anlage abhängig. Es fehlt im Säuglingsalter und Spielalter jene erst durch Erziehungs- und Arbeitsbedingungen geforderte t'bung des Herzens, bei der es im Schulalter zu den verschiedensten Störungen bel weniger Widerstandsfähigeren kommen kann. Mag dies einer der Gründe sein, weshalb das Kreislaufsystem des Säuglings bei ungewöhnlichen Ein- 
wirkungen wie Flüssigkeitsmangel, Gewichtsverlust, größerem Blutverlust, Kälte, Hitze. Infektionen, tieferen Narkosen oder narkotischen Giften schneller versagt als das der Frwachsenen. so spielt die primäre und ausschlaggebende Rolle dabei das GefäBsystem, die Regulation des Vasomotorenspiels der großen Gefäßgebiete. Das BlutgefäBsystem des Säuglings ist einer Reihe von Schädigungen gegenüber, auf die es gerade hier ankommt, wesentlich labiler als das des Erwachsenen. Und ich möchte Spitzy beipflichten. wenn er sagt. daß einmal stark gesunkene Herzkraft beim Kinde sich schwer wieder hebt, wenigstens in dem Sinne, daß dort, wo durch Vasomotorenschädigung der Blutdruck stärker gesunken ist, dieser sich schwer wieder herstellt und das Herz solche Störung auf die Dauer nicht kompensieren kann.

Es ist schon experimentell der deletäre Einfluß bekannt, den Flüssigkeitsmangel auf den Kreislauf besonders des wachsenden Tieres hat. Klinisch ist an die Kollapszustände zu erinnern, wie sie bei Verdurstung besonders junger, auch älterer Säuglinge aus den verschiedensten Ursachen entstehen können. Daß dabei zum Teil Infektionen mitwirken, kompliziert zwar die Beurteilung, aber der Ertolg der Wasserzufuhr, so bei Grippe und Nasopharyngitis mit Appetitlosigkeit (Göppert), Pyelitis, Ruhr (Göppert) erweist die entscheidende Bedeutung des Flüssigkeitsmangels für den Kreislauf des Säuglings in einem Grade, wie es beim Erwachsenen nicht, beim älteren Kinde nicht so früh der Fall ist. Göppert hat neuerdings an einer Reihe nicht mit Infektionen zusammenhängender klinischer Symptomenbilder, wie dem persistierenden Erbrechen bei schweren Magendarmerkrankungen, dem mit Zyanose einhergehenden Kollaps der Neugeborenen bei ungenügenden Trinkmengen an der Brust, die Wirkung der Wasserspeisung auf den bedrohten Kreislauf demonstriert. Bekannt sind ferner die Zusammenbrüche der Kreislauffunktion bei größeren Gewichtsstürzen. Dabei handelt es sich um mehr weniger akute Schwankungen der Widerstände in dem großen Gefäßgebiet der Haut oder auch um begleitende Lähmungserscheinungen im Splanchnikusgebiet. Hebung des Blutdrucks durch Digitalis nützt nichts, Koffein hilft bisweilen, ausschlaggebend ist aber die Flüssigkeitszufuhr.

Daß größere Blutverluste vom Säugling nicht ertragen werden, ist jedem Chirurgen bekannt. Es fragt sich, ob dabei mehr der qualitative Verlust für Sauerstoffaufnahme und Stoffwechsel oder schon der quantitative Flüssigkeitsverlust und der Kreislaufkollaps ursächlich bedeutungsvoll 1st. Ich nehme das letztere an. Denn schon bei mäßigen Verlusten kann ein gleiches dann der Fall sein, wenn infolge der Art 
der Narkose oder einer Abkühlung, zentral oder peripher bedingt, der Gefäßtonus geschwächt ist.

Den Einfluß der Kälte auf den Kreislauf des Säuglings kennen wir besonders aus Erfahrungen an Neugeborenen und Frühgeborenen. Bekannt ist ferner die lebensgefährliche Wirkung der Abkühlung der Bauchorgane bei Laparotomien; Eventration und ungenügende Wärmezufuhr bedingen beim Säugling einen stets tödlichen Kollaps infolge Splanchnikuslähmung. Dem Verfall des Kreislaufs ist das Herz nicht mehr gewachsen. Der Einfluß der Hitze auf die Säuglingssterblichkeit beruht letzten Endes auch auf einer Schädigung des Kreislaufs, die durch Ernährungsstörungen und Wasserverlust eingeleitet, durch Wassermangel unterhalten und gesteigert und zum Teil akut durch direkte Hitzeschädigungen bedrohlich wird.

Auch bei den Infektionen spielt im Säuglingsalter zentrale oder periphere Vasomotorenschädigung eine besondere Rolle; es besteht jedoch dem Erwachsenen gegenüber ein wesentlicher Unterschied nicht. Dagegen ist die Empfindlichkeit des Vasomotorenzentrums narkotischen Giften gegenüber wohl zweifellos erheblich größer als beim Erwachsenen, und es ist dies der Grund, weshalb das junge Kind eine tiefere Narkose schwer erträgt. Ist das Zentrum einmal geschädigt, so ist jeder Versuch, das Herz durch Exzitantien zur Überwindung des Kreislaufverfalles zu bringen, nutzlos. Die Frage, ob in einzelnen Fällen das Trauma der Operation oder die Narkose den Ausgang verschuldet, wird doch wohl dadurch entschieden, daß auch bei kleineren Eingriffen unsachgemäße Narkose gleich tiefergreifend wirken kann, wenn auch selbstverständlich unverhältnismäBiger Blutverlust oder toxische Vasomotorenschädigung durch die Grundkrankheit oder Abkühlung stets Momente sind, welche die zentrale Narkosevergiftung noch durch die periphere Schwächung des Kreislaufs unheilvoll komplizieren müssen.

Als Ursachen dafür, daß sich das Gefäßsystem des Säuglings diesen verschiedenen Schädigungen gegenüber labiler verhält, läßt sich, außer der besonderen Empfindlichkeit des Vasomotorenzentrums gegen Toxine und Gifte, vielleicht auch das beschriebene Verhältnis von Körpermaße zu Oberfläche anführen, zugleich mit der Größe des Splanchnikusgefäßgebietes, wobei den Schwankungen der Blutverteilung auch größere Ausschläge und Ansprüche an das Herz entsprechen können und endlich die Tatsache des Wasserreichtums des Säuglingskörpers in seiner Bedeutung als Regulator für die normalen Widerstände im Kreislauf, demzufolge Gewichtsverlust und Wassermangel immer auch auf Gefäßtonus und Herz zurückwirken. 
Ich bin danach der Uberzeugung, daß dem Kreislaufsystem des Säuglings bei weitem nicht das zugemutet werden darf, was beim Erwachsenen ohne Schaden möglich ist; und ich habe schon zu gleicher Zeit mit den für die Indikationen zu chirugischen Eingriffen oben mitgeteilten Grundsätzen eine Reihe weiterer Bedingungen für Narkose und Nachbehandlung durchzuführen begonnen. Es handelt sich dabei außer um die Narkose um Wärmeschutz und Sicherung der Flüssigkeitszufuhr.

Was die Narkose angeht, so bin ich der Uberzeugung, daß für Säugling und Kleinkind jede über das analgetische Stadium hinausgehende Narkose, jedes typische Narkosestaditum mit Erlöschen der Reflexe, jeder etwas tiefere Dauerzustand zu verterfen ist. Es schädigt oft nach kurzer Dauer das Vasomotorenzentrum. Erlaubt ist ausschließlich der beim Erwachsenen, als Narkose-Rausch bezeichnete Zustand leichter Betäubung und Schmerzempfindungslosigkeit, der beim Säugling so überaus leipht mit wenigen Tropfen zu erreichen und unter prinzipieller Unterbrechung der Zufuhr immer wieder durch einige Tropfen zu unterhalten ist. Ich benutze dazu eine kleine immer-ventilierte Maske oder nur einen Stiltupfer, der mit etwas Narkotikum beschickt vor Nase und Mund hin und her bewegt die Atemluft mit verdünnten Mengen desselben anreichert. Eine dauernde Narkose wie die Äthertropfnarkose (Spitzy) lehne ich ab. Nach meiner darauf gerichteten Beobachtung ist es bei dieser Methode gleichgültig, ob man mit Äther oder mit Chloroform betäubt; es gibt keinen Unterschied im Verlauf, auch keine Gefahren; Narkosenkomplikationen sind mir dabei unbekannt. Nur bei infektiösen Prozessen, bei denen eine toxische Vasomotorenschädigung vorausgesetzt werden kann, ziehe ich ausschließlich Äther vor.

Wo irgend möglich, kann die lokale Anästhesie (mit 0,5\% Novokain) Verwendung finden; das gleiche gilt von der Leitungsanästhesie. Die Lumbalanästhesie wird auch von den besten Kennern (Bier, Reh m) für das Kindesalter nicht empfohlen.

Sehr wichtig ist weiter der Wärmeschutz, die Verhütung der $\mathbf{A b}$ kühlung während der Operation. Es sind dazu anliegende weiche Flanellhüllen, heizbare Tische, elektrische Wärmekissen. Thermophore, Wärmkrüge oder erwärmte Sandsäcke zu empfehlen, ferner in der Umgebung des Operationsfeldes häufig zu wechselnde sterile warme Kompressen und Tücher. Auch nach beendigter Operation ist auf Wärmeschutz zu achten. Kälte oder Eisapplikation zu irgend einem Zweck sind nicht angebracht. 
Die dritte Forderung, die Sicherung der Flüssigkeitszufuhr in jedem Fall, setzt zunächst die bewußte Verhütung des Blutverlustes durch jede mögliche Maßnahme voraus. Die reguläre Ernährung soll keine oder eine Unterbrechung durch höchstens eine Mahlzeit erleiden. Die Nahrungsmengen bedürfen beim ernährungsgesunden Säugling kaum einer Einschränkung, man kann aber gut während der ersten 24 Stunden durch Einschiebung einer Nachtmahlzeit die einzelne Portion verkleinërn. Für den ganz jungen und den ernährungsgestörten Säugling kann die souveräne Bedeutung der Frauenmilch nicht oft genug betont werden. Während für die Ernährung der Ausfall von 1-2 Mahlzeiten zugestanden werden kann, darf die Flüssigkeitszufuhr niemals eine Unterbrechung erleiden und mu $B$ in jedem Fall durchgeführt werden. Ich halte es aber für notwendig, in der Nachbehandlung nach jedem etwas größeren Eingriff, insbesondere auch nach solchen bei infektiösen Prozessen einen Utberschuß an Flüssigkeit zuzuführen zur Ergänzung der Verluste, zur Unterstützung des erhöht beanspruchten Stoffwechsels und zur Sicherung der Kreislauffunktion. Es können dazu einmalige oder wiederholte kleme Mengen Ringerlösung subkutan oder durch Mastdarmirrigation, auch Tee mit Mühlbrunnen benutzt werden; be1 schwerer Appetitlosigkeit muß auch zu Mageneingießungen gegriffen werden.

Auf die zahlreichen Einzelfragen der Nachbehandlung soll bier nicht eingegangen werden. Sie sind so sehr von der verschiedenen Tèchnik abhängig, daß sie dem persönlichen Ermessen überlassen bleiben können. Nur weniges Allgemeine sei betont. Die Nachbehandlung soll so einfach wie möglich gestaltet werden. Es soll Rücksicht genommen werden auf die Agilität und motorische Betätigung und ihren Einfluß auf das Wohlbefinden des Kindes und deshalb jede nicht absolut notwendige Fesselung vermieden werden. Andererseits muß Beschmutzung und Infektion verhütet werden, und so sind von Schedes Vertikalsuspension bei Beinfrakturen ab bis heute zahlreiche Vorschläge gemacht worden. Als Wundverbände bevorzuge ich stets die einfachen flachen, eng die Wunde begrenzenden, mit Mastisol, Pflaster oder Kollodium fixierten und vermeide Watte und Binde. Tamponade wende ich so gut wie niemals an wegen ihrer Schmerzhaftigkeit, Infektionsund Nekrosegefahr. Bei ausgedehnten Wunden oder Abszessen am Rumpf ist häufigerer Wechsel steriler Mullwindeln oder Freilagerung in einer Schwebe oft der einzige, auch die Schmerzen des Verbandwechsels möglichst einschränkende Ausweg. Stets und besonders beim exsudativen Kind ist Hantpflege wichtig. Jede feuchte Wundbehandlung, schon 
an sich beim Säugling nicht ratsam, ist zu vermeiden; jedenfalls sollten feuchte Verbandtelle niemals dirckt auf die Haut gelegt werden.

\section{Spezielle Indikationen.}

Aus den dargelegten allgemeinen. Grundsätzen lassen sich die Gesichtspunkte für die Entscheidung des Einzelfalles zum Teil schon ableiten. Es soll hier noch, ohne Absicht auf Vollständigkeit, eine Reihe schwebender Fragen besprochen werden, wobei eine Einleitung der Operationsindikation nach ihrer Notwendigkeit in drei Gruppen zugrunde gelegt ist. $\mathrm{Zu}$ der ersten gehören die dringlichen lebensnotwendigen, zu der zweiten die gebotenen, $d$. h. solche, deren Vornahme zu normaler Entwicklung und Erziehung förderlich und früher oder später notwendig ist, zu der dritten die dex intern-chirurgischen Grenzgebiete; hierher sind alle die Erkrankungen zu rechnen, deren interne Behandlung Erfolg haben, deren chirurgische Behandlung aber irgendwann dringlich werden kann.

Zu der exsten Gruppe der dringlichen Operationen gehören zunächst die Spaltbildungen des Kopfes und der Wirbelsäule, deren operative Heilung je nach Ausdehmung aussichtsvoll oder unmöglich ist; überwiegend häufig, wenn auch nicht immer, schlieBt sich an die Heilung später die Bildung eines Hydrozephalus an, wodurch die Prognose in allen Fällen eine zweifelhafte ist. Es gehören hierher ferner die unvollständigen Ferschlüsse und die Stenosen am Magendarmkanal. Für die Bauchspaltbildungen (Nabelschnurbrüche) habe ich in einer neueren kasuistischen Zusammenstellung dargelegt, daß neben den echten Entwicklungshemmungen ursächlich mit gleichem Endergebnis mechanische Momente in Frage kommen. Die Prognose richtet sich nach dieser Atiologie und nach der Größe des Defektes und ist daher keineswegs immer sicher zu geben. Von den Stenosen sind der VerschluB des Anus oder des Rektums zu nennen, wobei die tieferliegenden und ausgedehnteren (als Obliterationen) größere Eingriffe erfordern und eine schlechtere Prognose haben. Die Anlegung eines Anus praeter naturalis ist eine aussichtlose Operation beim Säugling. Ungünstiger liegt es auch bei der operativen Behandlung der Dünndarmstenosen, nicht zum mindesten wegen der diagnostischen Schwierigkeiten.

Hierher zu rechnen sind ferner die akuten Darmverschlüsse durch Invagination und Brucheinklemmung. In beiden Fällen ist die frühe Erkennung von größter Wichtigkeit. Die bei Magendarmerkrankungen und bei Frühgeburten nicht seltenen, früh erkannten interkurrenten Brucheinklemmungen, die im Kaiserin Auguste Victoria Haus beobachtet 
wurden, waren ausnahmslos reponierbar. Die rechtzeitige Feststellung ist Bedingung. Aber auch poliklinisch habe ich häufiger Einklemmungen reponieren können, die 12 und mehr Stunden bestanden haben konnten. Bei stärkerer Peristaltik infolge Darmerkrankung ist die Aussicht geringer. Im Anschlu $B$ an die Reposition kann ich die Anlage des von Göppert angegebenen Bindenverbands auch aus eigener Anwendung empfehlen. Die Prognose der Operation des eingeklemmten Bruches wird häufig dadurch getrübt, daß die Dauer des Bestehens unsicher ist. Die Peritonitis des eingeklemmten Bruchsackes greift beim Säugling schneller auch auf das Peritoneum der Bauchhöhle über als beim Erwachsenen und ohne zuverlässige diagnostische Anzeichen. Die Invagination ist im Säuglingsalter überaus häufig (zirka $30 \%$ aller Fälle); die Dickdarminvagination, prognostisch die günstigere, ist glücklicherweise auch die häufigere. Alles hängt für den Erfolg der Behandlung von der Frühdiagnose ab. Akute Anfälle von Koliken, gelegentlich fühlbarer Tumor oder deutlich lokalisierte Schmerzhaftigkeit über dem Abdomen, bei Dickdarminvagination, Schleim-Blutabgang, bei sonst bestehender Verstopfung weisen auf die Diagnose hin. Die internen Behandlungsmethoden (Massage, Luft- oder Wasserfüllung) verdienen in jedem Fall versucht zu werden, jedenfalls innerhalb der ersten 12-24 Stunden und besonders ber den leichter zugänglichen Invaginationen des unteren Darmabschnittes mit fühlbarem Tumor. Gelingt die Lösung nicht, so ist die Laparotomie alsbald anzuschließen; von einer frühzeitigen Operation ist doch wenigstens in der Hälfte der Fälle Heilung zu erwarten; eine Prognose ist $1 \mathrm{~m}$ Einzelfall kaum zu stellen, da es sich auch darum handelt, ob ein größerer oder kleinerer Eingriff ausreicht. Die selteneren und wenig aussichtsvollen Ileusformen können übergangen werden.

In allen diesen Fällen handelt es sich um überwiegend schwere Eingriffe z. T. an Neugeborenen, und es gelten ganz besonders die für die Narkose, Nachbehandlung und Ernährung aufgestellten Forderungen. Anzuschließen wären noch die primären infektiösen Prozesse, akute Phlegmone, isolierte Osteomyelitis mit und ohne Gelenkbeteiligung, die nicht auf Allgemeinerkrankung oder Allgemeininfektion beruhen und die auch bei Brustkindern vorkommen. Sie sind im ganzen selten im Vergleich zu den sekundären, auf Herabsetzung der Immunität beruhenden Infektionen, die ich zu den Erkrankungen der Grenzgebiete rechnen möchte. Hier hat die frühzeitige Operation eine vorwiegend bessere Prognose und ist auch, da es sich um akute Prozesse handelt, notwendig. Auch die Heilung ist hier eine schnellere und selten komplizierte. 
In die zweite Gruppe der gebotenen Operationen gehören in der Hauptsache die angeborenen Bildungsfehler, deren Beseitigung für die bessere körperliche Entwicklung und Ausbildung wünschenswert ist. Es sind dabei noch einige offene Fragen, die hier nur aufgezeigt und nicht erledigt werden können, weil die Erfahrungen über die eine oder andere Methode, konservative orthopädische oder chirurgische Behandlung, frühere oder spätere Operation zu einer Entscheidung nicht ausreichen.

So stehen in der Frage des Zeitpunktes der Operation der Hasenscharte sich die Ansichten noch schroff gegenüber. Riedel hat aus seinem größeren chirurgischen Material gelegentlich mitgeteilt, daß er niemals im ersten Lebenshalbjahr, meist erst mit dreiviertel Jahren operiert hat, andere wie Spi tzy bekennen sich zur frühzeitigen Operation, unter Voraussetzung des Mindestgewichtes und gesunden Zustandes in den ersten Monaten. Kinder mit mäßigen Spaltbildungen können, wie es auch bei uns beobachtet wurde, bei gutgeleiteter Stilltechnik, die Brustwarze mit ihrem großen Munde weit und sicher fassen, so daß sie mit Erfolg bis zu einem späteren Termin gestillt und in widerstandsfähigerem Zustand operiert werden können. Größere Hasenscharten mit Gaumenspalten sind durch die Möglichkeit stomachaler und katarrhalischer Infektionen gefährdet. Ich selbst habe nie im ersten Lebensvierteljahr, die gefährdeten im zweiten, den Rest im dritten und vierten Lebensvierteljahr operiert. Ein oft schwer zu überwindender Widerstand geht von den Eltern aus; hier hat die Fürsorge aufklärend zu wirken. Die Operation der Gaumenspalte ist neuerdings, wie erwähnt wurde, von Helbing schon im Säuglingsalter mit vorzüglichem Erfolg durchgeführt worden, während die übrigen Chirurgen nicht vor dem zweiten Jahre operieren. Unter seinen 283 Fällen sind 100 operierte Säuglinge sämtlich geheilt.

Auch für die Behandlung der Leistenbrüche besteht keine übereinstimmende Auffassung. Für die konservative Methode leistet im ersten Jahr das Wollbruchband Ausgezeichnetes; im Spielalter setzt dann eine gutdurchgeführte Bruchbehandlung ein mit einer Pelotte, die flach und weich gepolstert die Atrophierung des Gewebes ausschließt. Uber den Umfang einer Dauerheilung nach diesein Verfahren besteht keine ausreichend festgelegte Erfahrung. Göpp’ert schätzt sie auf etwa $80 \%$. Häufig ist die notwendige Konsequenz in der Behandlung schwer zu erreichen. An der Möglichkeit der Dauerheilung vermag ich nach eigenen Beobachtungen nicht zu zweifeln. Spitzy bestreitet sie. Bei größeren Brüchen ist die Radikaloperation etwa vor der Schulzeit 
unter günstigen Bedingungen ratsam. Die prinzipielle frühzeitige Operation hat bei Erfolg den Vorzug, daß alle Schwierigkeiten der Bruchbehandlung wegfallen, aber die Dauerresultate sind doch je nach der Technik recht verschieden und keineswegs Rezidive bei Operation im Säuglingsalter weniger möglich als später: Soziale Verhältnisse werden den Operationstermin mitbestimmen müssen. Dort, wo die notwendige Pflege und Sorgfalt fehlt, wird man sich früher zur Operation entschließen. Ich selbst habe in solchen Fällen im dritten, seltener im zweiten Jahr operiert, aber stets exakte Pfeilernaht gemacht. Einen früheren Termin halte ich nicht für berechtigt.

Nabelbrüche sind nach den Erfahrungen der Kinderärzte in einer überwiegenden Zahl mit konservativer Methode mit Erfolg zu behandeln. Greifbare kasuistische Angaben über Dauerresultate fehlen auch hier. Die relative Seltenheit der Nabelbrüche beim Erwachsenen spricht für den Erfolg. Die Reposition und die Verhinderung des die Heilung störenden Drucks der Eingewède ist wesentlich leichter zu überwinden,

Auch für Hydrozele ist die frühzeitige Operation nicht notwendig. eine Obliteration nach Rückgang des Ergusses ist zweifellos möglich. Doch kann man bei Komplikation von Leistenbruch mit Hydrozele zunächst den letzteren mittels der kleinen Radikaloperation beseitigen, um die Resorption des ersteren zu erleichtern.

Die Notwendigkeit, das, was man mit dem Sammelnamen Phimose bezeichnet, im Säuglingsalter zu operieren, wird von der Pädiatrie mit Recht nicht mehr anerkannt. Die vielfachen, z. T. auf Laienvorstellung beruhenden Begründungen, wie Behinderung der Miktion oder Stuhlentleerung, oder ungünstiger Einfluß auf bestehenden Leistenbruch, sind hinfällig. Indessen ist es doch nicht richtig, epitheliale Verklebung und Phimose gleichzustellen, denn es kann nicht bestritten werden, daß es echte Phimosen gibt; es fehlt die eindeutige Abgrenzung für die Diagnose; jedenfalls kommt aber im Säuglingsalter die Operation nicht in Frage. In seltenen Fällen können spasmophile Krampfzustände am Sphincter vesicae zu Miktionsstörungen führen. Auch sei erwähnt, daß ich in vereinzelten Fällen als Ursache von Miktionsstörungen, einmal auch mit Zystitis, an die sonst stets zu denken ist, kompliziert, nicht eine Phimose, sondern ęine angeborene Verengerung der Harnröhre feststellen konnte; es besteht eine Faltenbildung meist am Ubergang zur Pars pendula. Die operative Korrektur des Epispadie und Hypospadie gehört in die spätere Kindheit.

Hierher gehören weiter die angeborenen Verbildungen der Muskulatur und des Skeletts. Bei der Indikation zur Operation steht die 
schon besprochene Tatsache der gesteigerten Deformierung mit dem Wachstum im Vordergrund und die Möglichkeit einer frühzeitigen Korrektur durch orthopädische Maßnahmen oder relativ kleinere Eingriffe. Ein Schiefhals, der zunächst sich nur auf die Verbildung eines Muskels oder einer kleinen Gruppe bezieht und durch eine kleine Diszision in Lokalanästhesie in seiner Ursache behoben werden kann, führt unbehandelt zur Deformierung der Hals- und Rückenwirbelsäule und zum Zurückbleiben der Muskulatur der gleichen Seite. Ein Klumpfuß, der die Folge einer Verkürzung der Achillessehne und of $t$ sehr geringfügigen Verbildungen des Mittelfußes ist, wird schon im zweiten bis dritten Lebensjahre zu einer schwer reparablen MiBbildung, abgesehen davon, daß das Laufen unmöglich ist. Hier empfiehlt sich frühzeitig in den ersten Wochen beginnend eine modellierende Massage und Verband: behandlung, durch die häufig die Deformität völlig behoben und eine spätere Operation überflüssig wird. Ähnliches gilt für Plattfuß und Hackenfuß. Bel der kongenitalen Hüftluxation kann bei sorgfältiger Frühdiagnose, die wohl noch oft übersehen wird; mit Recht auch die frühzeitige Behandlung durchgeführt werden. Die Resultate der Pfannenbildung sind, soweit man das nach dem noch geringen Material beurtelen kann, sehr günstige. Zur Fixation habe ich abnehmbare nach dem Körper modellierte Pappschienen verwandt, wobei Hautpflege und Bad möglich gemacht ist.

Auf Einzelheiten der Fragen des Zeitpunktes der Behandlung der rachitischen Verbildungen soll nicht eingegangen werden. Die allgemeinen Grundsätze der pädlatrischen Behandlung, die Im Vordergrund stehen müssen, sind schon hervorgehoben.

Als letzte, dritte Gruppe lassen sich die Erkrankungen der. internchirurgischen Grenzgebiete zusammenfassen. An erster Stelle steht hier immer die pädiatrische Behandlung, weil einerseits die Erkrankungen Folgen und Komplikationen interner Leiden sind und deshalb ihre frühzeitige Erkennung von besonderer Bedeutung ist, anderseits weil auch ihre Behandlung im frühen Stadium mit Erfolg eine interne ist. Indessen kann es mangels frühzeitiger Diagnose und Behandlung, und unter ungünstigen Umständen zu einem lebenbedrohenden Zustand kommen und es ist deshalb die Frage des Zeitpunktes der Dringlichkeit des chirurgischen Eingreifens ebenso wesentlich, wie sie je nach den persönlichen Erfahrungen und dem ärztlichen Standpunkt kontrovers ist.

Als Repräsentanten der nichtinfektiösen Erkrankungen dieser Gruppe sind der Pylorospasmus und de Hirschsprungsche Krankheit zu nennen. Es sollen hier nicht die klinischen Fragen der Pathogenese 
und Pathologie des Pylorospasmus, auch nicht Einzelheiten der konservativen Behandlung wie die Diätetik und die Pylorussondierung u. a. erörtert werden, um so mehr da sie auf Grund der Erfahrungen des Hauses in dieser Festschrift schon behandelt werden. Neben den Erfolgen der klinischen Behandlungsmethoden. bleibt noch eine kleinere Anzahl Fälle, bei denen die operative Behandlung notwendig wird. Die Höhe des Gewichtsverlustes im Verhältnis zum Anfangsgewicht (die Questsche Zahl) darf dazu nicht allein den Ausschlag geben. Wichtiger ist das Fortschreiten der Abnahme bei längerer Behandlung, der Hungerstuhl, das Versiegen der Urinsekretion und die verminderte Immunität, also ausgesprochene Zeichen einer Störung des inneren Stoffwechsels. Die Operationsmethode von Weber-Ramstedt bedeutet einen wesentlichen Fortschritt für die Vereinfachung des notwendigen Eingriffs und für dessen Prognose. Weber hatte gegenüber den früher üblichen eingreifenden plastischen Verfahren mit Eröffnung des Magens und Naht oder der viel zu komplizierten Gastroenteroanastomose, mit der einfachen Schnittdurehtrennung der Serosa. und Muskularis des verdickten Pylorus unter Erhaltung der Schleimhaut auch ohne nachfolgende Naht vollen Erfolg. Rietschel gibt die Erfolge der von Weber operierten Fälle, im ganzen 19; auf $84,2 \%$ an.

Auch für die Hirschsprungsche Krankheit ist die Auffassung ihrer funktionellen Pathogenese für die interne Therapie fruchtbar geworden. Sie ist durch Mitteilungen von Göppert, Pfisterer, Fütterer und Mitteldorpf u. a. klargestellt worden. Um so früher die Diagnose gesichert ist, um so weniger stellen sich die schwereren sekundären Veränderungen des Dickdarms ein. Meteorismus, unter Umständen sichtbare Peristaltik bei auffallender Stuhlverhaltung weisen auf die Diagnose hin, die durch digitale Exploration gesichert werden kann. Göppert ließ das die Abknickung überwindende Darmrohr mit Erfolg und ohne Schaden monatelang liegen, in anderen Fällen läßt er täglich, wie auch andere Pädiater, das Darmrohr zúr Entleerung und Spülung einführen. Für die chirurgische Behandlung kommen nur vorwiegend große und prognostisch zweifelhafte Operationsmethoden in Frage, es ist also von der internen Behandlung alles, was sie leisten kann, zu versuchen.

Den Hauptanteil an den Erkrankungen der Grenzgebiete haben die infektiösen Prozesse. Dazu gehören einmal die große Zahl jener sekundären Infektionen, die in der Hauptsache der Ausdruck einer gesunkenen Immunität sind, nicht so selten aber auch die Folge unsachgemäßer Pflege und Vernachlässigung oder Unterschätzung der ersten 
Krankheitszeichen und primären Infektionsherde. Es sind die Pyodermien, die Furunkulose, die anschließenden phlegmonösen Prozesse, die Drüsenabszesse, manche Fälle von Mastoiditis im Gefolge der Otitis media, der Retropharyngealabszeß, die Abszedierung der Mastitis, die sekundären Knochen- und Gelenkeiterungen. In allen diesen Fällen ist, wie schon hervorgehoben wurde, die interne Behandlung, die Hebung der Immunität durch Besserung des Ernährungszustandes der erste Grundsatz der Therapie. Der chirurgische Eingriff sei so klein und schonend wie möglich, soweit dabei die Beherrschung der Eiterung und die Verhinderung weiterer Propagierung gesichert ist. Bei dem großen Material des Hauses wurden bei zahlreichen Fällen von Otitis media nur zwei Fälle von Mastoiditis beobachtet. Für sehr ausgedehnte Furunkulosen empfehle ich die Eröffnung mit einem messerförmigen Thermokauter, wobei Blutung, Verbreitung. des Eiters auf die Haut und Wiederverklebung des Schnittrandes vermieden wird. Aus der Zahl der ernsteren infektiösen Erkrankungen des Grenzgebietes sei das Empyem hervorgehoben. Hier liegen die offenen Fragen, wie sie auch bei anderen infektiösen Prozessen bezüglich Diagnose und Therapie in der Kontroverse der Meinungen bestehen, klar zutage. Sie beginnen schon mit den Schwierigkeiten der Diagnose hinsichtlich der Frage, ob ein Empyem postpneumonisch oder durch Sekundärinfektion entstanden oder septisch ist, hinsichtlich der Dauer des Bestandes und der Ausdehnung der Affektion; Fragen, die für Prognose und Wahl der Behandlung entscheidend sein können. Wo die oft großen differentialdiagnostischen Schwierigkeiten nicht lösbar sind, besteht auch der Pessimismus der chirurgischen Behandlung gegenuiber nicht zu Recht. Dort, wo die Frühdiagnose beherrscht wird, können viele kleinere Empyeme durch einfache Punktion, größere durch Rippenresektion zur sicheren Heilung gebracht werden. Manche Exsudate, auch kleine Empyeme bilden sich spontan zurück. Die Erfahrungen über die Häufigkeit der primär serösen Ergüsse, wie sie Ylppö aus dem Material des Kaiserin Auguste Victoria Hauses mitgeterlt hat, kann ich bestätigen. Septische Empyeme zu operieren, bleibt auch unter günstigen Ernährungss- und Pflegebedingungen ein Spiel mit einer letzten Möglichkeit. Der Erfolg darf nicht auf Kosten der Operation gebucht werden. Was die Methode betrifft, so läßt sich die Buelau sche Drainage doch wohl nur für länger bestehende, schwach infektiöse, fast fieberlose Empyeme rechtfertigen. Für die größere Zahl bleibt die Rippensekretion die gegebene Methode auch für den Säugling. Auf die Technik kommt alles an; Wärmeschutz, Art der Narkose, Art und Tempo der Entleerung und schonende Nachbehandlung mit beson- 
derer Bərücksichtigung der Lungenaffektion, soweit sie noch vorhanden, und der Immunität sind entscheidend.

\section{Literaturverzeichnis.}

1. Bertling, Über die Verwendbarkeit des Urethans in der Kinderheilkunde. Berl. klin. Wochenschr. 49, 147. 1912.

2. Blühdorn, Untersuchungen über die therapeutisch wirksame Dosierung von Kalksalzen. Monatsschr. f. Kinderheilk. 12, 195. 1913.

3. Czerny·Keller, Des Kindes Ernährung, Ernährungsstörungen usw. Wien 1906/1917 (Deuticke).

4. Ehrlich, Beiträge zur experimentellen Pathologie und Chemotherapie 1909.

5. Finkelstein, Lehrbuch der Säuglingskrankheiten. Berlin 1905/1912 (Fischer).

6. Fütterer und Mitteld orpf, Ein Fall von großem kongenitalem Divertikel der Flexura sigmoides. Virchows Archiv 106, 555. 1886.

7. Göppert, Die therapeutische Verwendung der Kalksalze. Med. Klinik 10, 1003.1914.

8. - Nasen-, Rachen-, Ohrerkrankungen des Kindes. Berlin 1914 (Springer).

9. - Die einheimisohe Ruhr im Kindesalter. Ergeb. d. inn. Med. u. Kinderheilkunde. 15, 180. 1917.

10. - Therapeutische Vorschläge ans dem Gebiet der Kinderheilkunde. Therap. Monatsh. 29, 511. 1915.

11. - Therapeutische Vorschläge aus dem Gebiet der Kinderheilkunde. Therap. Monatsh. 30, 574. 1916.

12. - Über einen Fall von angeborener Abknickung des Dickdarms in Rücksicht auf die sog. angeborene Dilatation und Hypertrophie. Arch. f. Verdauungskrankh. 5, 175. 1899.

13. - Ventilverschlufs durch Abknickung im untersten Teil des Dickdarms im späteren Kindesalter. Berl. klin. Wochenschr. 49, 588. 1912.

14. Grünfelder, Beeinflussung der Magensaftsekretion durch Infektion. Zeitschrift f. exper. Pathol. u. Therap. 16; 141. 1914.

15. Helbing, Diskussion in der Berliner medizinischen Gesellschaft. Ref. Berl. klin. Wochenschr. 21, 509. 1918.

16. Langstein und Meyer, Säuglingsernährung und Säuglingsstoffwechsel. 2. Aufl. Berlin 1914 (Springer).

17. Langstein, Ernährung und Wachstum der Frühgeborenen. Berl. klin. Wochenschr. 52, 631. 1915.

18. - Eigentümlichkeiten und Ernährung der Neugeborenen (einschließlich der Frühgeborenen). Jahresk. f. ärztl. Fortb. 7. Juni 1916.

19. - Die Ernährungsstörung im Säuglingsalter. Theorie und Praxis. Arbeiten aus dem Kaiserin Auguste Victoria Hause. Berlin 1919 (Springer).

20. Pfisterer, Obstipation infolge Darmabknickung. Jahrb. f. Kinderheilk. 65, 160. 1907.

21. Reiche, Welches sind die Lebensaussichten der vorzeitig geborenen Kinder? Therap. Monatsh. 30, 392. 1916.

22. Rietschel, Zur operativen Behandlung der angeborenen Pylorusstenose des Säuglings. Med. Klinik 19, 457. 1918. 
Walther Usener: Indikationen für chirurg. Eingriffe im Säuglingsalter. 103

23. Rott, Zur Frnährungstechnik frühgeborener Säuglinge. Zeitschr. f. Kinderheilk. 5, 134. 1912.

24. Spitzy, Handbuch der Kinderheilk. (Pfaundler-Schloßmann). 5. Bd.

25. - Chirurgische und orthopädische Eingriffe im Säuglingsalter. Verhandlg. d. Gesellsch. f. Kinderheilk. Wien 1913.

26. Thie mich, Lehrb. d. Kinderheilk. (Feer), Jena (Fischer).

27. Thomas und Hornemann, Experimentelle Beiträge zur Frage der Beziehungen von Infektion und Ernährung. Verhandl. d. Gesellsch. f. Kinderheilk. Wien 1913 u. Biochem. Zeitschr. 57, 456/473. 1913.

28. Usener, Über Nabelschnurbruch. Jahrb. f. Kinderheilk. 77, 181. 1913.

29. Weigert, Über den Einfluß der Ernährung auf die chemische Zusammensetzung des Organismus. Jahrb. f. Kinderheilk. 61, 178. 1905.

30. Ylppö, Zur Klinik und Cytologie der Pleuraergüsse. Zeitschr, f. Kinderheilk. 17, 169. 1918. 